Research Article

\title{
Use of Acoustic Emission for the Detection of Brittle Rock Failure under Various Loading Rates
}

\author{
Hai-qing Shuang, ${ }^{1,2}$ Shu-gang Li, ${ }^{1,2}$ Lang Liu $\mathbb{D}^{2,3}$ Gao-feng Chen, ${ }^{1,2}$ and Ki-Il Song $\mathbb{B}^{4}$ \\ ${ }^{1}$ School of Safety Science and Engineering, Xi'an University of Science and Technology, Xi'an, Shaanxi 710054, China \\ ${ }^{2}$ Key Laboratory of Western Mine Exploitation and Hazard Prevention with Ministry of Education, Xi'an, Shaanxi 710054, China \\ ${ }^{3}$ School of Energy Engineering, Xi'an University of Science and Technology, Xi'an, Shaanxi 710054, China \\ ${ }^{4}$ Department of Civil Engineering, Inha University, Incheon 402-751, Republic of Korea \\ Correspondence should be addressed to Ki-Il Song; ksong@inha.ac.kr
}

Received 9 November 2017; Accepted 9 January 2018; Published 8 March 2018

Academic Editor: Hang Lin

Copyright () 2018 Hai-qing Shuang et al. This is an open access article distributed under the Creative Commons Attribution License, which permits unrestricted use, distribution, and reproduction in any medium, provided the original work is properly cited.

\begin{abstract}
Acoustic emission has a direct correspondence to the internal damage of a material. To determine the effects of the loading rate on the mechanical properties of rock, the initial damage was characterized using the acoustic emission technique when a uniaxial preloading was imposed on a cylindrical rock sample. On this basis, the uniaxial compression test was conducted on sandstone that contains initial damage induced under a range of loading rates. The effects of the initial damage and loading rate on the mechanical properties of rock were analyzed. The uniaxial preloading generated randomly distributed microcracks in the natural rock. The results showed that the acoustic emission and positioning technique can characterize accurately the damage and its position due to preloading. The development of microcracks was found to be strongly dependent on the loading rate. Moreover, the loading rate accelerated the degradation of the rock strength. The effects of the loading rate and initial damage on the mechanical properties of rock are a complicated coupled process. From the experimental test result, a constitutive equation was constructed based on the damage mechanics.
\end{abstract}

\section{Introduction}

In recent years, the number of high-strength mining faces in Western China has increased. High-strength mining refers to a coal mining method with the mining intensity of fully mechanized top-coal caving and a large mining height, which has a high propulsion speed, large stope size, and high backstopping efficiency. The difference in the advance speed of the working face affects not only the transfer process of rock stress but also the speed of the loading and unloading processes. The probability of disaster induced by rock failure increases as the advancing speed increases in a working face [1]. In addition, it was reported based on field monitoring data that rapid excavation at the working face can cause serious surface subsidence.

To examine the relationship between the advancing speed of the working face and the rock stress transfer mechanism, basic experimental studies have been carried out in the laboratory due to many difficulties related to onsite research. The advancing speed of the working face can be considered as the loading rate on a laboratory scale. The loading rate has a significant influence on the deformation characteristics and failure mechanism of rock [2]. Li et al. [3] examined the growth of microcracks at different loading rates. Liang et al. [4], Liu et al. [5], Zhou et al. [6], Zhang et al. [7], and Kodama et al. [8] studied the effects of the strain rate on the mechanical properties of rock. Li et al. [9-11] analyzed the effect of the loading rate on the nonlinear mechanical properties of coal and suggested an efficient method to evaluate the impact performance of coal.

In addition, Daphalapurkar et al. [12], Lin et al. [13], and Cao and Lin [14] examined the effect of microcracks on the tensile properties of hard and brittle materials by numerical simulations. Yang et al. [15] conducted an experimental study on natural rock containing preexisting fissures using an "artificial customization technique." Yang et al. [16, 17] 


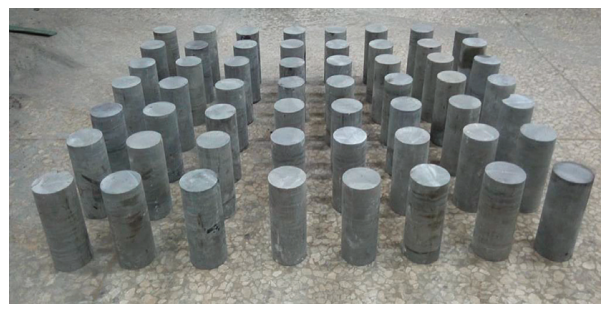

FIgURE 1: Sandstone samples for the experimental test.

TABLE 1: Grouping of the sandstone samples.

\begin{tabular}{|c|c|c|c|c|c|c|}
\hline Group number & Sample number & Mass $(\mathrm{kg})$ & Height (mm) & Diameter (mm) & $\begin{array}{l}\text { Longitudinal wave } \\
\text { velocity }(\mathrm{km} / \mathrm{s})\end{array}$ & $\begin{array}{c}\text { Loading rate } \\
(\mathrm{mm} / \mathrm{min})\end{array}$ \\
\hline \multirow{4}{*}{ Group 1} & $\# 2$ & 0.53 & 101.4 & 49.25 & 4.67 & 0.03 \\
\hline & $\# 3$ & 0.53 & 101.3 & 49.18 & 4.67 & 0.05 \\
\hline & $\# 6$ & 0.53 & 102 & 49.15 & 4.67 & 0.07 \\
\hline & $\# 7$ & 0.53 & 101.5 & 49.38 & 4.67 & 0.09 \\
\hline \multirow{4}{*}{ Group 2} & $\# 1$ & 0.52 & 100.1 & 48.88 & 4.67 & 0.03 \\
\hline & $\# 5$ & 0.52 & 100.4 & 48.65 & 4.67 & 0.05 \\
\hline & $\# 8$ & 0.52 & 100.3 & 48.81 & 4.67 & 0.07 \\
\hline & $\# 9$ & 0.52 & 100.8 & 48.55 & 4.67 & 0.09 \\
\hline
\end{tabular}

and Zhang et al. [18] examined the effects of the shape and quantity of damage-induced fissures on the mechanical properties of brittle materials, of which regularly distributed initial fissures had been prefabricated artificially. In general, the damage-induced fissures in brittle materials are distributed in the form of open cracks and apertures, and microcracks are distributed randomly in a natural rock mass. Chen [19] and Wang et al. [20] produced damageinduced initial fissures within the rock samples by preloading. They used an ultrasonic longitudinal wave velocity for quantitative characterization of the initial damage. Based on previous studies, uniaxial preloading is imposed on a brittle rock sample to generate initial damage in the form of microcracks in this study. In particular, the initial damage was characterized quantitatively using an acoustic emission technique. The distribution of microcracks was analyzed visually using the acoustic emission positioning technique. Furthermore, a uniaxial compression test was conducted on the intact rock samples and the rock samples containing initial damage at a range of loading rates to determine the effect of the loading rate and initial damage on the mechanical properties of rock. From the experimental test result, a constitutive equation was constructed based on the damage mechanics.

\section{Design of the Test Prototype}

2.1. Sample Preparation. The rock type used in the present study was sandstone obtained from the Liuhuanggou Coal Mine, Yankuang Xinjiang, China. Quartz, ankerite, and anorthose are the main rock-forming minerals of the sandstone. The average longitudinal wave velocity and average density of sandstone were $4.4 \mathrm{~km} / \mathrm{s}$ and $2800 \mathrm{~kg} / \mathrm{m}^{3}$, respectively. The sandstone samples were prepared, $500 \times 1000 \mathrm{~mm}$ (diameter $\times$ length), in accordance with the recommendation suggested by the International Society for Rock Mechanics. To ensure the homogeneity of the sandstone samples, all samples were drilled from an intact sandstone block, and the sound wave velocity was obtained. Only the sandstone samples with the same wave velocity and a stable waveform were selected for further experimental testing. This means that the sandstone samples were intact and did not have notable fissures inside. Figure 1 shows the part of selected rock samples. Sandstone samples were separated into two groups, as shown in Table 1: Group 1 for an evaluation of the loading rate without preloading (i.e., no initial damage) and Group 2 for an evaluation of the loading rate after preloading with initial damage.

2.2. Testing Method. Acoustic emission (AE) is a physical phenomenon of transient elastic waves produced by the rapid release of internal elastic energy due to rapid deformation and crack growth of hard and brittle materials when they are subjected to external or internal forces [21]. Acoustic emission has a direct correspondence to the internal damage of a material. The damage level of a material can be estimated from the accumulative number of acoustic emission events because the new damage must be accompanied by the occurrence of acoustic emission [22]. In addition, knowledge of the fracture mechanics shows that the fracture of hard and brittle materials is caused by internal defects. The distribution of internal defects governs the failure behavior of a brittle material and its mechanical properties. The acoustic emission technique can be used effectively to detect the generation of internal defects due to loading and its positioning.

In this study, the internal damage of rock due to preloading was characterized quantitatively by the accumulative number of acoustic emission events, and the location of 


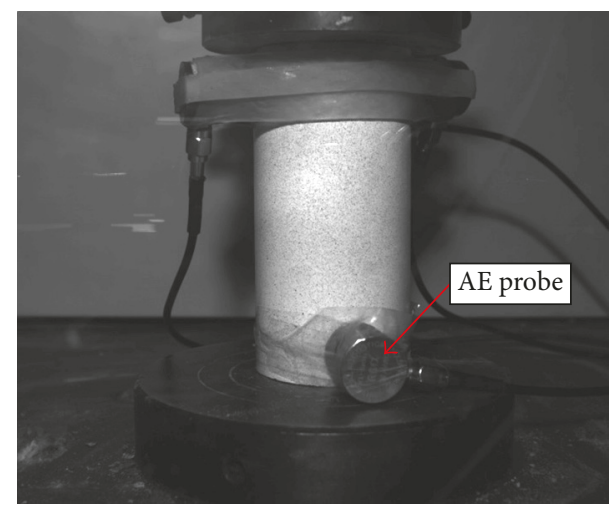

Figure 2: Use of acoustic emission for the detection of microcracks.

microcracks was analyzed using the positioning technique. As shown in Section 2.1, sandstone samples in Group 2 were preloaded to produce the initial damage. A slow loading rate and maximum force were maintained to ensure the full development of fissures within the rock during preloading. Preloading was performed at a loading rate of $0.2 \mathrm{kN} / \mathrm{s}$ and a maximum force limited to $<200 \mathrm{kN}$. The loading rate was maintained for $3 \mathrm{~min}$. The uniaxial compression test at different loading rates was conducted on the intact rock samples without preloading and the samples containing the initial damage due to preloading. Four different loading rates (i.e., $0.03,0.05,0.07$, and $0.09 \mathrm{~mm} / \mathrm{min}$ ) were selected, as shown in Table 1 . Four acoustic emission probes were attached to the rock specimen, as shown in Figure 2.

\section{Detection of Initial Damage with Acoustic Emission}

3.1. Positioning of Microcracks with Acoustic Emission. The occurrence of the acoustic emission event is due mainly to the generation and growth of microcracks. Transient monitoring of the location where the acoustic emission event occurred can reflect the progressive evolution of the fracture. The spatial distribution of microcracks, the direction, and spatial curved surface shape of crack growth within the rock samples can be observed visually. Figure 3 presents the location map of the acoustic emission events for Group 2 during preloading.

Figure 3 shows that AE events are concentrated at both ends of the rock samples and that the initial damage caused by preloading is distributed evenly within the rock samples, excluding the AE events caused by friction around $20 \mathrm{~mm}$ of the upper and lower ends. The preloading is a useful tool for simulating the distribution of internal microcracks within a rock specimen.

3.2. Quantitative Characterization of Initial Damage. The longitudinal wave velocity and accumulative number of acoustic emission events can be used to characterize the level of rock damage quantitatively. In this study, the longitudinal wave velocity was obtained from the Group 2 samples containing microcracks due to preloading before and after loading. The longitudinal wave velocity and acoustic emission test results (excluding the AE events around $20 \mathrm{~mm}$ of the upper and lower ends) were compared to select a more accurate characterization method for the initial damage. The results are summarized in Table 2.

Table 2 shows that the accumulated number of acoustic emission events produced by the four rock samples during the preloading process is $12 \sim 14$, and the decrease in longitudinal wave velocity is minor. Interestingly, the more the accumulated number of $\mathrm{AE}$ events, the greater the change in the longitudinal wave velocity. This suggests that the $\mathrm{AE}$ event accompanies the increase in the number and density of microcracks and causes a significant reduction of the longitudinal wave velocity.

Variance analysis was conducted based on the test results to evaluate the validity among the accumulated number of $\mathrm{AE}$ events and longitudinal wave velocity as an indicator for better quantitative analysis. The variance is the degree of deviation from the average and is used to measure the fluctuation size of a batch of data (i.e., the size of the data deviation from the average number), expressed as $S^{2}$. When the number of specimens is identical, a greater variance indicates larger data fluctuations. This also implies that the validity of the indicator becomes increasingly unstable as the variance increases. The variance can be calculated as follows:

$$
S^{2}=\frac{1}{n-1} \sum_{1}^{n}\left(X_{i}-\bar{X}\right)^{2},
$$

where $n$ is the number of samples, $X_{i}$ is the individual, and $\bar{X}$ is the average of the sample. The normalization should be adopted for variance analysis to consider the inconsistency of data. The variance of the normalized data can be calculated as follows:

$$
S_{1}^{2}=\frac{S^{2}}{\bar{X}^{2}}=\frac{1}{n-1} \sum_{1}^{n}\left(\frac{X_{i}-\bar{X}}{\bar{X}}\right)^{2} .
$$

The variances of the accumulated number of AE events and the longitudinal wave velocity were 0.006 and 0.357 , respectively. Therefore, the deviation obtained from the $\mathrm{AE}$ test was smaller than that of the longitudinal wave velocity. Hence, the AE test can be considered to be a good quantitative characterization method for initial damage.

\section{Brittle Rock Failure at Various Loading Rates}

4.1. Stress-Strain Curve. Figures 4 and 5 show the stressstrain curves obtained from Groups 2 and 1, respectively.

The rock samples undergo a compaction stage, elastic deformation stage, elastoplastic deformation stage (not obvious), and postpeak failure stage during uniaxial compression loading (Figures 4 and 5). The rock samples of Group 1 have similar stress-strain curves regardless of the loading rate because the stress and strain increase steadily until the stress falls suddenly due to brittle rock failure. The rock samples of Group 2 with initial damage showed stress adjustment at a slow loading rate of $0.03 \mathrm{~mm} / \mathrm{min}$. The stress adjustment disappeared as the loading rate was increased. In addition, the stress decreased suddenly due to brittle rock failure. On the other hand, the strain at failure of Group 2 was slightly larger 


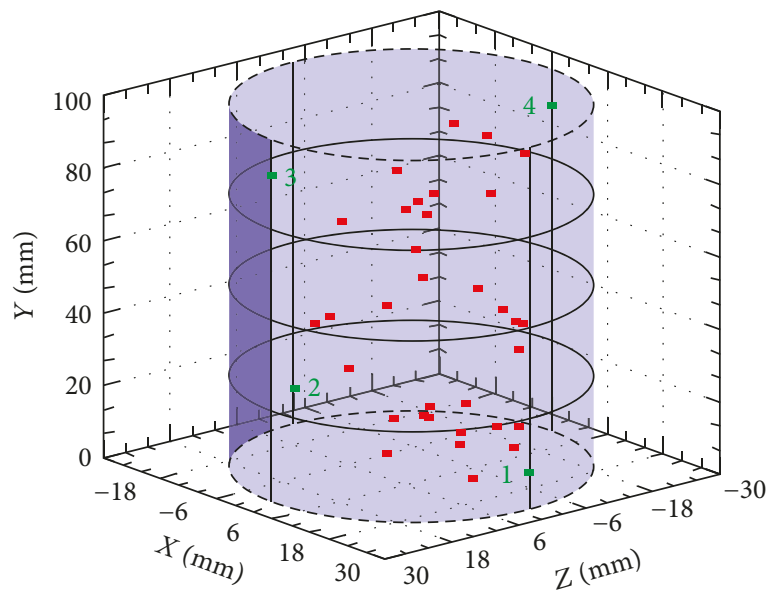

(a)

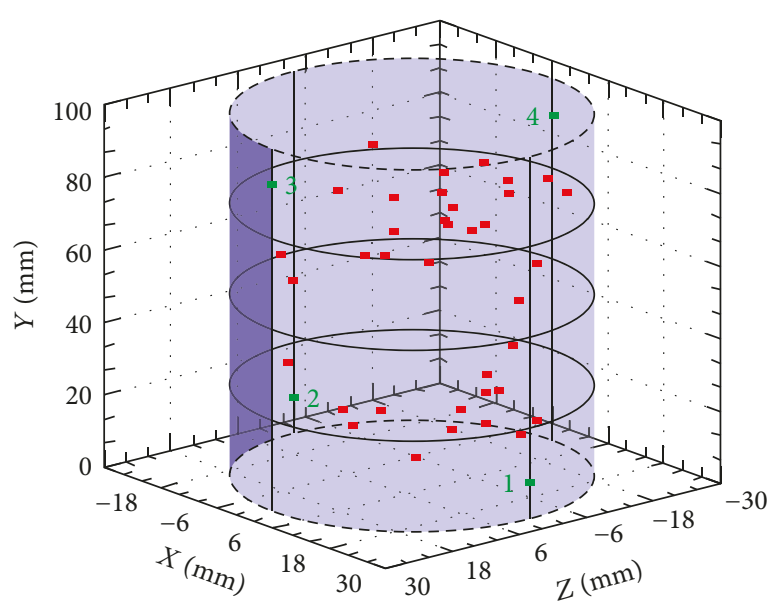

(c)

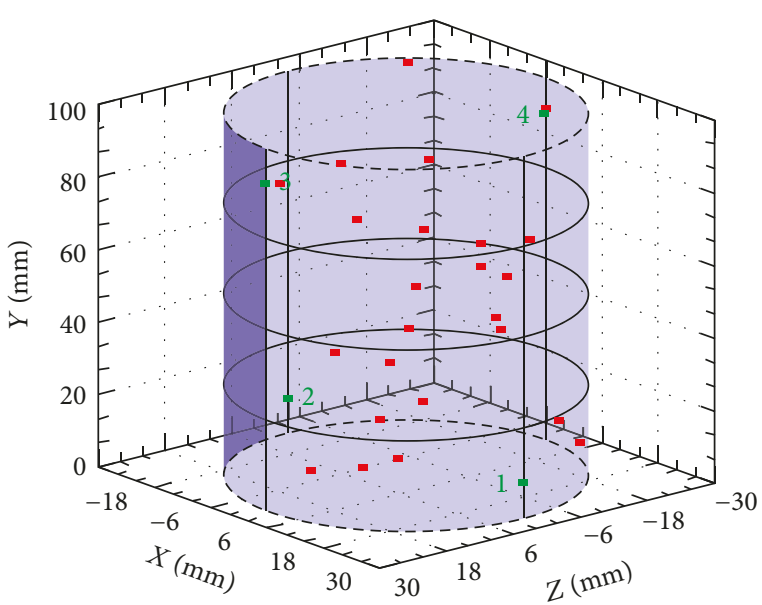

(b)

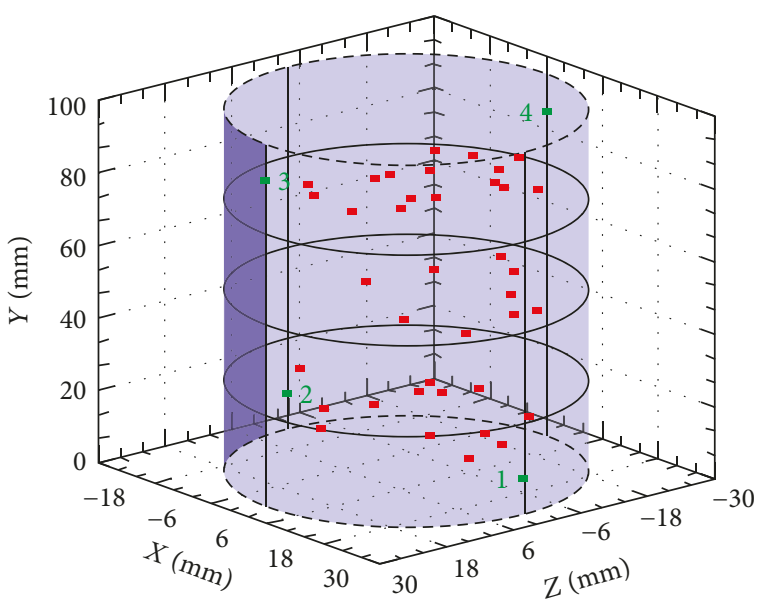

(d)

FIgURE 3: Location map of acoustic emission events. (a) Location map for the initial damage events of rock sample \#1; (b) location map for the initial damage events of rock sample \#5; (c) location map for the initial damage events of rock sample \#8; (d) location map for the initial damage events of rock sample \#9.

than that of Group 1 under the same loading rate. This suggests that the initial microcracks extend as the stress level increases, and a macroscopic fracture surface forms within the samples. In particular, the relatively large strain at failure of Group 1 can be caused by slipping of the macroscopic fracture surface within the rock samples when the loading rate is low.

\subsection{Analysis for Intensity Characteristics. Figure 6 shows} the relationship between the loading rate and uniaxial compressive strength of the original rock samples without damage and rock samples with initial damage.

The uniaxial compressive strength increased with increasing the loading rate for both groups (Figure 6). For original rock samples, the UCS obtained from a minimum loading rate was approximately $77.4 \%$ of that obtained from the maximum loading rate. For rock samples with initial damage, the UCS obtained from the minimum loading rate was approximately $59 \%$ of that obtained from the maximum loading rate. The reason for the above phenomena can be understood by the rock composed of the physical medium and damage
TABLE 2: AE events and reduction of the wave velocity due to preloading.

\begin{tabular}{lcc}
\hline $\begin{array}{l}\text { Sample } \\
\text { number }\end{array}$ & $\begin{array}{c}\text { Accumulative } \\
\text { number of AE } \\
\text { events during } \\
\text { preloading }\end{array}$ & $\begin{array}{c}\text { Reduction of } \\
\text { wave velocity } \\
\text { due to preloading } \\
(\mathrm{km} / \mathrm{s})\end{array}$ \\
\hline$\# 1$ & 13 & 0.20 \\
$\# 5$ & 12 & 0.09 \\
$\# 8$ & 14 & 0.43 \\
$\# 9$ & 12 & 0.14 \\
\hline
\end{tabular}

defects wrapped by the physical medium. When the loading rate was slow, the energy dissipated mainly for fissure growth, and the rock samples showed low compressive strength at a low loading rate, resulting in a fully developed fissure. As the loading rate increases, the fissures cannot develop fully, and microcracks do not interconnect due to the rapid deformation. In such cases, the energy is stored mainly in the physical medium in the form of elastic energy, which increases the uniaxial compressive strength of the rock samples. 


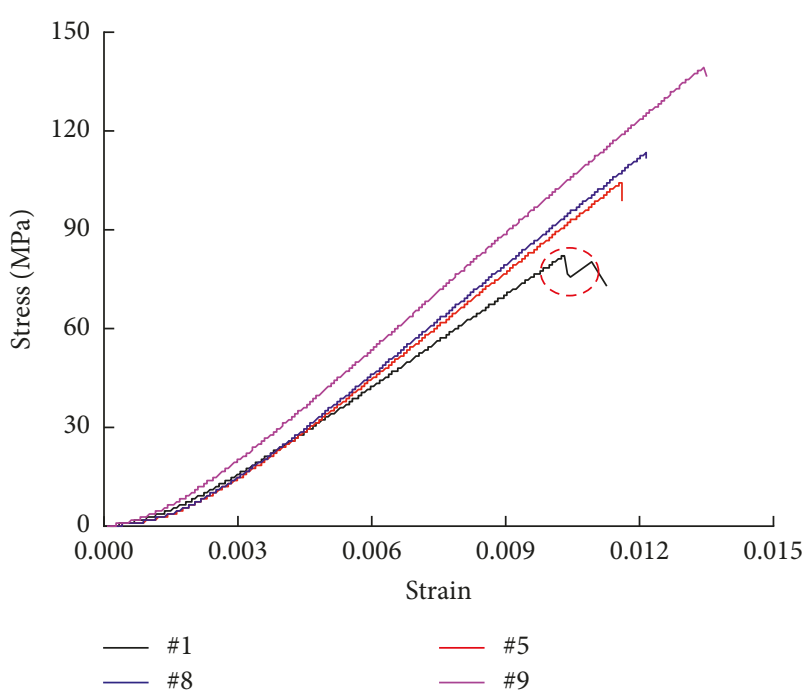

FIgURE 4: Stress-strain curve of Group 2 with initial damage.

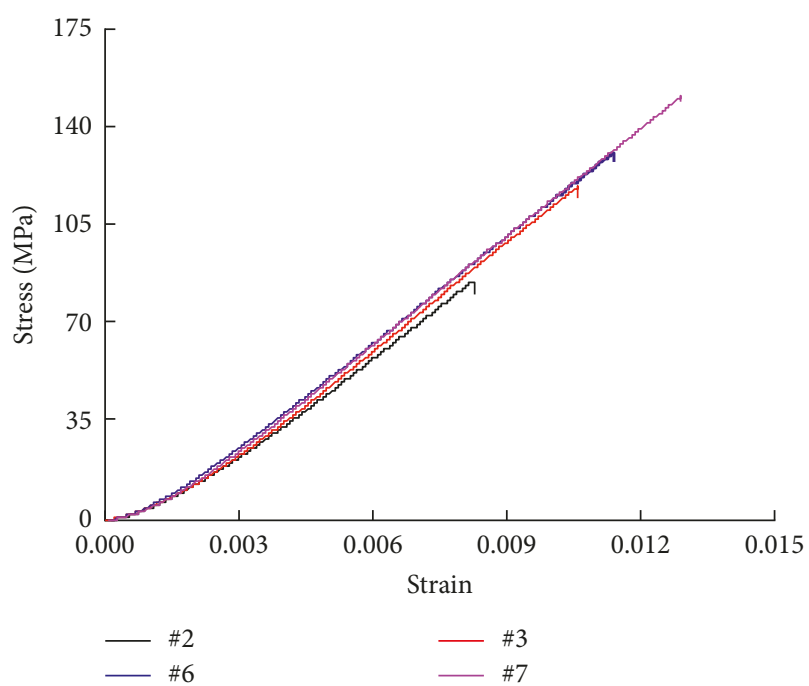

FIgURE 5: Stress-strain curve of Group 1 without initial damage.

The uniaxial compressive strength can be reduced due to the initial microcrack (Figure 6). The compressive strengths of the rock samples containing microcracks are lower than those of the original rock samples. The deterioration effect of the initial damage on the compressive strength differed according to the loading rates. For a quantitative description of the deterioration effect of the initial damage on the strength of a rock sample, the deterioration rate is defined as follows:

$$
K_{\sigma}=\frac{R_{c}-R_{\mathrm{cd}}}{R_{c}} \times 100 \%,
$$

where $K_{\sigma}$ is the deterioration rate, $R_{c}$ is the uniaxial compressive strength of the original rock sample $(\mathrm{MPa})$, and $R_{\mathrm{cd}}$ is the uniaxial compressive strength of the damaged rock sample. The attenuation range of the rock samples' bearing capacity increases with increasing deterioration rate. On the

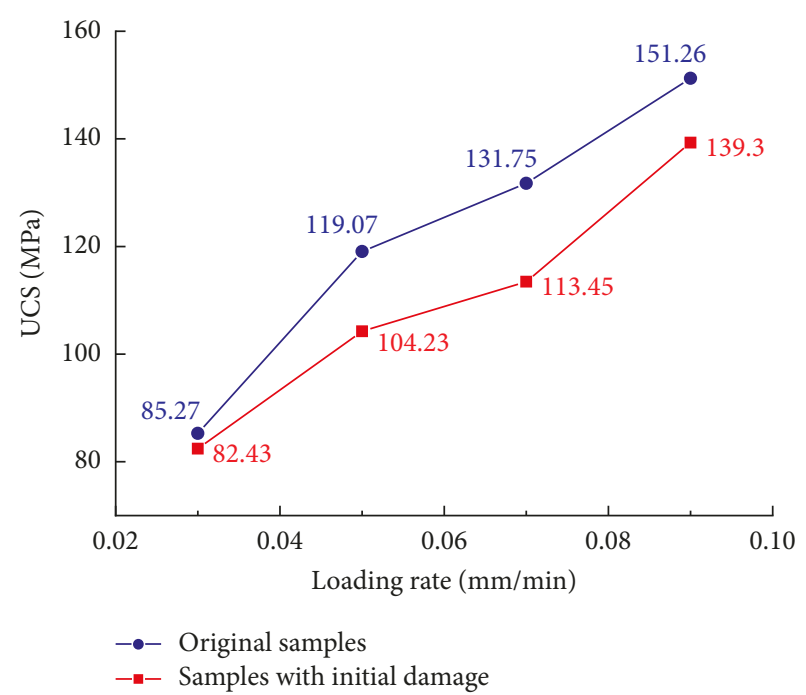

FIgURE 6: Relationship between the loading rate and uniaxial compressive strength (UCS).

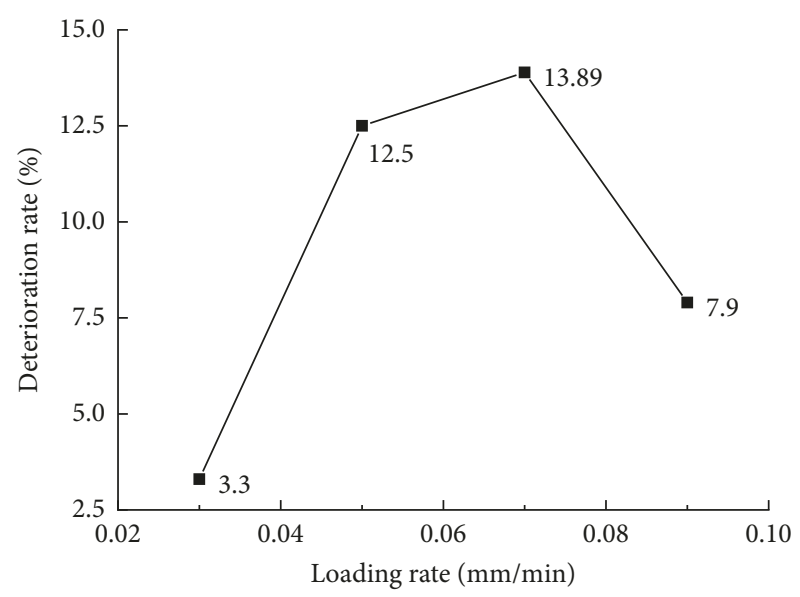

FIGURE 7: Relationship between the loading rate and deterioration rate.

other hand, the bearing capacity of the intact rock samples becomes similar with decreasing attenuation.

The deterioration rate was obtained using (3) depending on the loading rate, and the results are presented in Figure 7. When the loading rate was low, the initial damage showed a less deterioration effect on the rock samples. The deterioration rate increased with increasing loading rate. On the other hand, this phenomenon is not persistent; the deterioration rate decreases when the loading rate exceeds $0.07 \mathrm{~mm} / \mathrm{min}$. As the loading rate increases, the growth of microcracks is accelerated. Therefore, the deterioration rate increases with increasing loading rate until the loading rate is slow enough to develop microcracks fully. The deterioration rate decreases because microcracks cannot be fully developed due to the rapid loading rate.

4.3. Analysis for Deformation Characteristics. Figures 8 and 9 show the maximum axial strain and corresponding elastic 


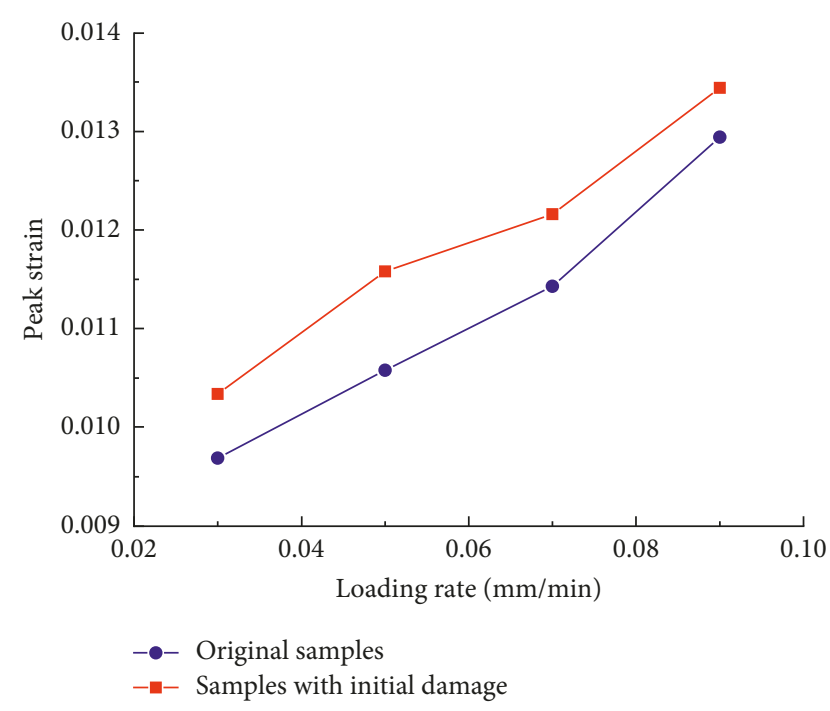

FIGURE 8: Relationship between the peak strain and loading rate.

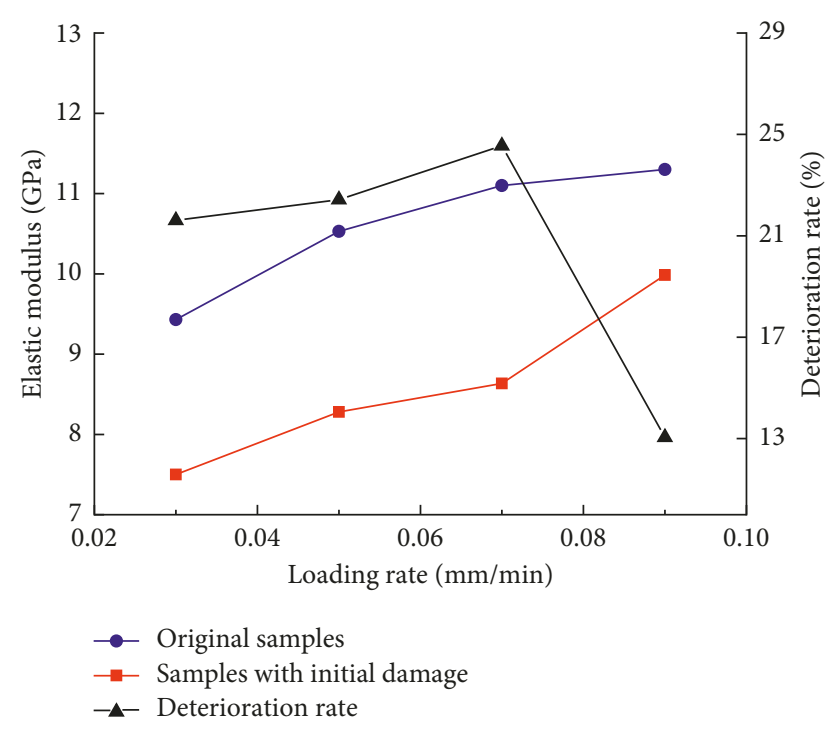

FIgURE 9: Change in the elastic modulus and deterioration rate depending on the loading rate.

modulus of the original rock samples and the rock samples with initial damage at different loading rates.

The maximum axial strain generally increases with increasing loading rate (Figure 8 ). When the loading rate is slow, slippage and dislocation are concentrated at the main crack area within the rock samples, and there is sufficient time for crack growth. In such cases, the rock samples only need a small deformation to produce a dominant macrocrack and damage. When the loading rate is increased, the mesoscopic defects within the rock samples have no time to form a dominant crack but deformation of the physical medium, so larger deformation is necessary to cause complete fracture in the rock samples.
The maximum axial strain of the rock samples with initial damage is larger than that of the original rock samples. The initial damage enlarges the compaction stage and shortens the elastic stage, leading to an increase in axial strain. This means that the initial damage can improve the ductility of rock.

The elastic modulus increased with increasing loading rate and converges for the original rock samples (Figure 9). The elastic modulus increased with increasing loading rate for the rock samples with initial damage. The nondeformability of the rock samples improved, and the elastic modulus increased. The difference in elastic modulus between the two samples decreased with increasing loading rate. This suggests that a faster loading rate weakens the deterioration effect. To observe more clearly the deterioration effect of initial microfissures depending on the loading rate on the elastic modulus of the rock samples, the deterioration rate of the elastic modulus can be obtained by replacing the compressive strength in (3) with the elastic modulus, as shown in Figure 9. The deterioration rate of the elastic modulus decreases significantly when the loading rate is high.

4.4. Analysis of the Fracture Mode. The loading rate and the initial damage have an important influence on the strength and strain of rock samples. The different strength performance corresponds to the different failure characteristics. Figure 10 shows the typical failure mode of rock samples due to uniaxial compression depending on the loading rate.

The loading rate has a significant effect on the failure mode of rock samples. When the loading rate was slow, the rock samples showed mainly splitting and stretch-draw fracture and two relatively large rock blocks formed after failure. As the loading rate increased, stretch-draw and shear fractures could be found, and the fracture intensity became severe. When the loading rate exceeded $0.07 \mathrm{~mm} / \mathrm{min}$, the rock samples were fractured completely in the form of rockburst, as shown in Figures 10(c) and 10(d). This is consistent with the theoretical derivation in the literature [3].

\section{Analysis for the Damage Mechanics of the Loading Rate Effect}

As for the simplest model, the rock can be considered an elastic medium with a homogeneous, isotropic nature so that the mechanical properties of rock are only related to the state of stress. When the state of stress is unchanged, the forms of deformation and fracture will not change depending on the loading rate. On the other hand, the physical medium of the rock material is composed of various rock-forming minerals that contain microcracks. In addition, the mechanical properties of the rock samples with initial damage are influenced heavily by the loading rate.

Based on the assumption that the intensity of the microbody follows the Weibull distribution for the addition of damage mechanics for a simple model, the probability density function becomes 


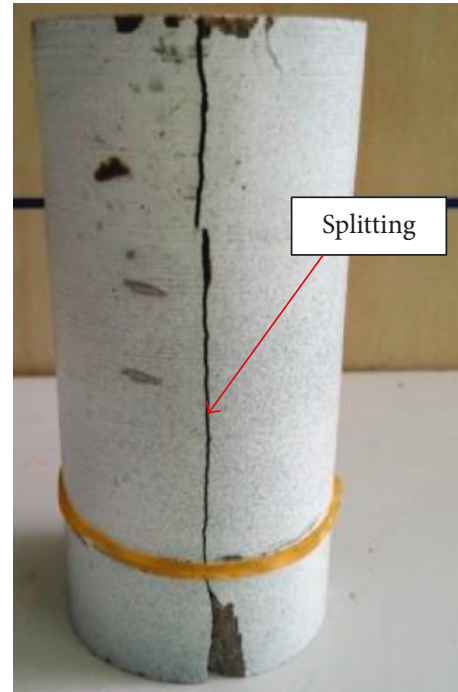

(a)

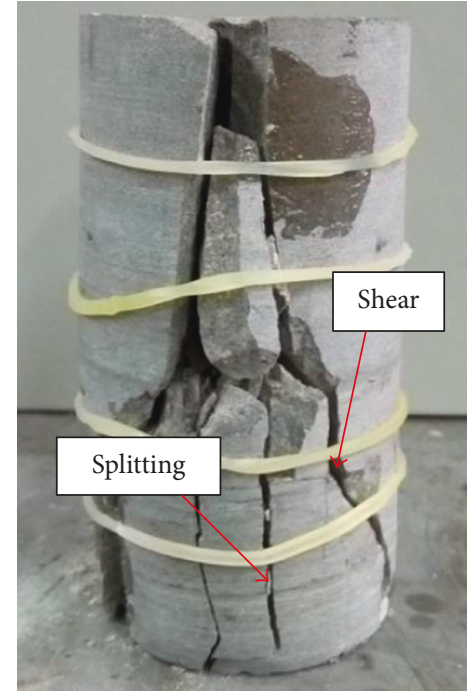

(b)

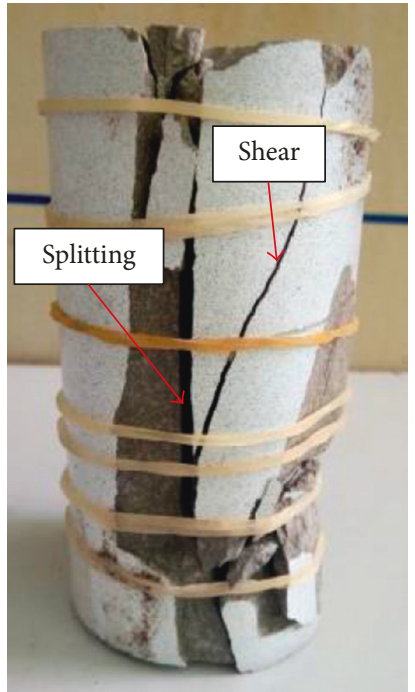

(c)

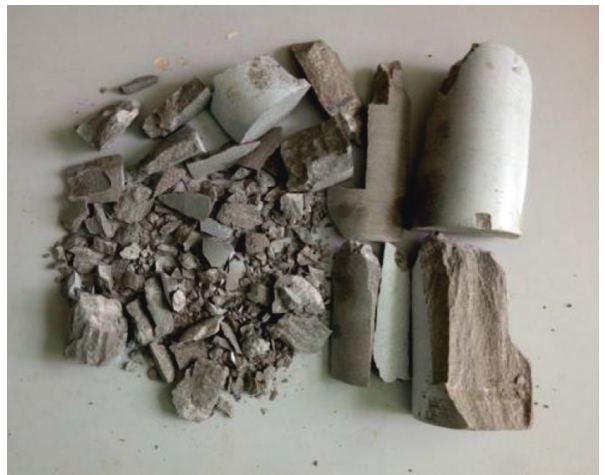

(d)

FIGURE 10: Failure mode of rock depending on the loading rate. (a) \#2 (loading rate $=0.03 \mathrm{~mm} / \mathrm{min}$ ), (b) $\# 4$ (loading rate $=0.05 \mathrm{~mm} / \mathrm{min}$ ), (c) $\# 6$ (loading rate $=0.07 \mathrm{~mm} / \mathrm{min}$ ), and (d) $\# 8$ (loading rate $=0.09 \mathrm{~mm} / \mathrm{min}$ ).

$$
f(x, \lambda, k)= \begin{cases}\frac{k}{\lambda}\left(\frac{x}{\lambda}\right)^{k-1} \exp \left[-\left(\frac{x}{\lambda}\right)^{K}\right], & x \geq 0, \\ 0, & x \leq 0\end{cases}
$$

where $\lambda$ is the scale parameter or the proportional parameter, $k$ is the shape parameter greater than 0 , and $x$ is the random variable.

If the random variable is characterized by the parameter time $t$ related to the loading rate, the physical meaning of the Weibull distribution is the probability of a microbody fracture when the loading time is $t$, and the damage variable $D$ can be calculated using

$$
D=\int_{0}^{t} f(x, t, k) d t=1-\exp \left[-\left(\frac{t}{t_{0}}\right)^{k}\right]
$$

From (5), the damage variable increases with increasing loading time due to the growth of microcrack defects within the rock mass and slippage under the action of force: the shorter the loading time (the faster the loading rate), the better the integrity of the rock sample. Based on the assumption of equivalent strain, the relationship between the stress and the loading time can be defined as

$$
\sigma=E \mathcal{E}(1-D)=E \varepsilon \exp \left[-\left(\frac{t}{t_{0}}\right)^{k}\right] .
$$

Equation (6) shows that the stress increases with decreasing loading time. This is the theoretical explanation for the increase in the compressive strength of rock with increasing loading rate.

In addition, from the point of view of energy, some external energy can be stored in the physical medium in the form of elastic energy, and some is dissipated in the form of fissure growth. As reported elsewhere [22], when the crack length is $\Delta L$, the energy consumption, $W_{\Delta L}$, is

$$
W_{\Delta L}=\frac{\pi(1-\nu) \sigma_{t}^{2} \Delta L^{2}}{8 G}
$$

where $\sigma_{t}$ is the tensile stress at the crack tip, which can be considered the inherent properties of a material. 
The deformation of rock samples is caused mainly by fissure growth when the loading rate is slow. Because the dissipation energy is large and the absorbed elastic energy is lower, the rock samples show a simple failure mode. The fissure growth within the rock becomes slow when the loading rate is large, and the deformation of the physical medium governs the behavior of the rock sample. The elastic energy of the rock also increases with increasing loading rate, and it will be released suddenly after the peak strength is reached. In such cases, the rock samples show more intensive fracture in the form of rockburst.

In recent years, the advancing speed at the working face is very fast due to fully mechanized coal mining technology. High-speed advances bring significant economic benefits but also have a negative impact. Therefore, it is important to understand the influence of the initial damage and loading rate on the mechanical properties of rock to prevent a disaster during mining.

\section{Conclusion}

To find a reasonable way to express damage, the characteristic by the number of acoustic emission events is used for the initial damage caused by rock precompression, and it is more accurate compared to the longitudinal wave velocity. The acoustic emission monitoring shows that the prepressed rock samples produce randomly distributed microfissure damage, which is consistent with the field.

The original rock samples and the rock samples with initial damage underwent a compaction stage, elastic deformation stage, plastic deformation stage, and postpeak fracture stage under different loading rates. The existence of initial damage makes the compaction and plastic deformation stages of the rock samples longer. On the other hand, an increase in loading rate makes the compaction stage and the plastic deformation stage of the rock samples shorter, and the rock shows a purely brittle fracture.

The strength and peak strain of the original rock samples and the rock samples with initial damage increase almost linearly with increasing loading rate, and the initial damage plays a deterioration role on the strength and elastic modulus of the rock samples. The deterioration effect increases with increasing loading rate when the loading rate is slow and decreases when the loading rate is increased further.

The effect of the loading rate and initial damage on the mechanical properties of rock is a complex coupling process, in which the loading rate is more significant. This study revealed failure of the rock samples from splitting fracture to stretch-draw and shear fractures with increasing loading rate and established a damage evolution equation considering the time effect. Therefore, it is important to understand the influence of the initial damage and loading rate on the mechanical properties of rock to prevent the disasters during mining.

\section{Conflicts of Interest}

The authors declare that they have no conflicts of interest regarding the publication of this paper.

\section{Acknowledgments}

This work was supported by the National Natural Science Foundation of China (nos. 51327007, 51674188, 51504182, 51304156, and 51674192), the Scientific Research Program Funded by Shaanxi Provincial Education Department (no. 15JK1466), and the project funded by China Postdoctoral Science Foundation (no. 2015M582685). This work was also supported by the National Research Council of Science and Technology (NST) grant by the Korea government (Ministry of Science, ICT and Future Planning) (no. CRC-16-02KICT).

\section{References}

[1] Y. Li, T. H. Yang, H. Liu, X.-G. Hou, and H. Wang, "Effect of mining rate on the working face with high-intensity mining based on microseismic monitoring: a case study," Journal of Geophysics and Engineering, vol. 14, no. 2, pp. 350-358, 2017.

[2] D. J. Xue, H. W. Zhou, and Z. H. Wang, "Failure mechanism and mining-induced mechanical properties of coal under different loading rates," Journal of China Coal Society, vol. 41, no. 3, pp. 595-602, 2016.

[3] J. Li, Q. P. Huang, and X. D. Ren, "Dynamic initiation and propagation of multiple cracks in brittle materials," Materials, vol. 6, no. 8, pp. 3241-3253, 2013.

[4] W. G. Liang, Y. S. Zhao, S. G. Xu, and M. B. Dusseault, "Effect of strain rate on the mechanical properties of salt rock," International Journal of Rock Mechanics and Mining Sciences, vol. 48, no. 1, pp. 161-167, 2011.

[5] J. X. Liu, W. Liu, and C. H. Yang, "Experimental research on effects of strain rate on mechanical properties of shale," Rock and Soil Mechanics, vol. 35, no. 11, pp. 3093-3100, 2014.

[6] H. Zhou, Y. S. Yang, and H. B. Xiao, "Research on loading rate effect of tensile strength property of hard brittle marble-test characteristics and mechanism," Chinese Journal of Rock Mechanics and Engineering, vol. 32, no. 9, pp. 1868-1875, 2013.

[7] X. P. Zhang, Q. Zhang, and S. Wu, "Acoustic emission characteristics of the rock-like material containing a single flaw under different compressive loading rates," Computers and Geotechnics, vol. 83, pp. 83-97, 2017.

[8] J. Kodama, T. Goto, Y. Fujiia, and P. Hagan, "The effects of water content, temperature and loading rate on strength and failure process of frozen rocks," International Journal of Rock Mechanics \& Mining Sciences, vol. 62, no. 2, pp. 1-13, 2013.

[9] H. T. Li, C. X. Jiang, and Y. D. Jiang, "Mechanical behavior and mechanism analysis of coal samples based on loading rate effect," Journal of China University of Mining \& Technology, vol. 44, no. 3, pp. 430-436, 2015.

[10] H. T. Li, L. Song, and H. W. Zhou, "Evaluation method and application of coal burst performance under the effect of loading rate," Journal of China Coal Society, vol. 40, no. 12, pp. 2763-2771, 2015.

[11] Q. Q. Liu, Y. P. Cheng, K. Jin, Q. Tu, W. Zhao, and R. Zhang, "Effect of confining pressure unloading on strength reduction of soft coal in borehole stability analysis," Environmental Earth Sciences, vol. 76, no. 4, p. 173, 2017.

[12] N. P. Daphalapurkar, K. T. Ramesh, L. Graham-Brady, and J.-F. Molinari, "Predicting variability in the dynamic failure strength of brittle materials considering pre-existing flaws," Journal of the Mechanics \& Physics of Solids, vol. 59, no. 2, pp. 297-319, 2011. 
[13] H. Lin, W. Xiong, and Q. X. Yan, "Three-dimensional effect of tensile strength in the standard Brazilian test considering contact length," Geotechnical Testing Journal, vol. 39, no. 1, pp. 137-143, 2016.

[14] R. H. Cao and H. Lin, "Experimental and numerical study of failure behavior and energy mechanics of rock-like materials containing multiple joints," Advances in Materials Science and Engineering, vol. 2017, Article ID 6460150, 17 pages, 2017.

[15] S. Q. Yang, C. H. Lv, and T. Qu, "Investigations of crack expansion in marble having a single pre-existing hole: experiment and simulations," Journal of China University of Mining \& Technology, vol. 38, no. 6, pp. 774-781, 2009.

[16] S. Q. Yang, Y. H. Dai, and L. J. Han, "Experimental study on mechanical behavior of brittle marble samples containing different flaws under uniaxial compression," Engineering Fracture Mechanics, vol. 76, no. 12, pp. 1833-1845, 2009.

[17] S. Q. Yang, Y. H. Huang, H. W. Jing, and X.-R. Liu, "Discrete element modeling on fracture coalescence behavior of red sandstone containing two unparallel fissures under uniaxial compression," Engineering Geology, vol. 178, pp. 28-48, 2014.

[18] Z. B. Zhang, E. Y. Wang, and D. Chen, "The observation of AE events under uniaxial compression and the quantitative relationship between the anisotropy index and the main failure plane," Journal of Applied Geophysics, vol. 134, pp. 183-190, 2016.

[19] Y. L. Chen, M. X. Dai, and M. L. Liu, "Experimental investigation on freezing damage characteristics of granite with initial damage," Chinese Quarterly of Mechanics, vol. 34, no. 1, pp. 74-80, 2013.

[20] Z. Q. Wang, E. C. Yan, and Y. J. Wang, "Generation of damage fracture system and indirect-quantitative characterization of fracture ratio to schist," Chinese Quarterly of Mechanics, vol. 36, no. 4, pp. 697-702, 2015.

[21] J. L. Pei, J. F. Liu, and J. P. Zuo, "Investigation on dynamic evolution process of natural fractures based on acoustic emission position," Chinese Journal of Rock Mechanics and Engineering, vol. 32, no. 4, pp. 696-704, 2013.

[22] C. A. Tang, Catastrophe in Rock Unstable Failure, Coal Industry Publishing House, Beijing, China, 1993. 


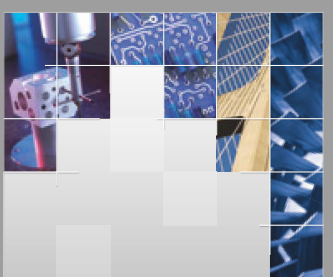

\section{Enfincering}
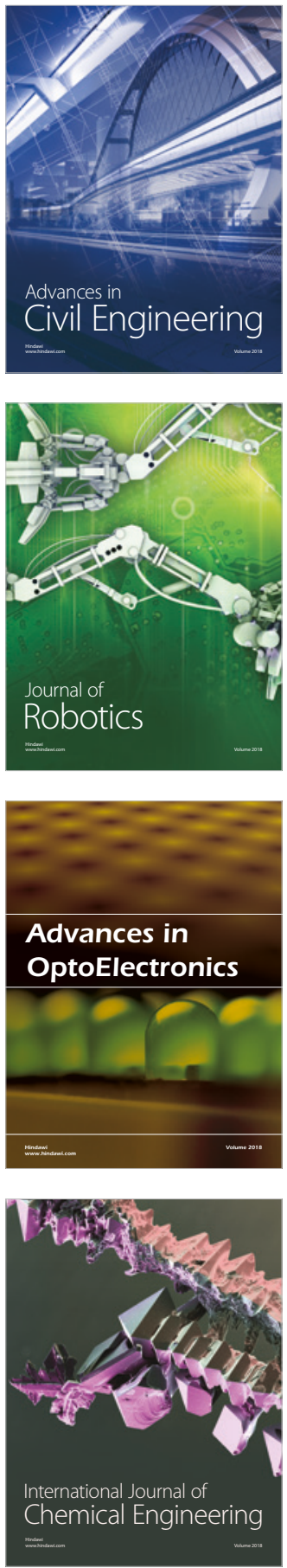

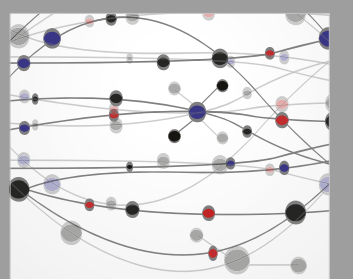

\section{Rotating \\ Machinery}

The Scientific World Journal

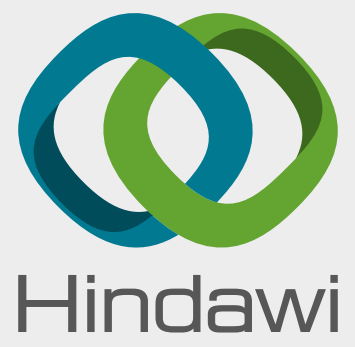

Submit your manuscripts at

www.hindawi.com
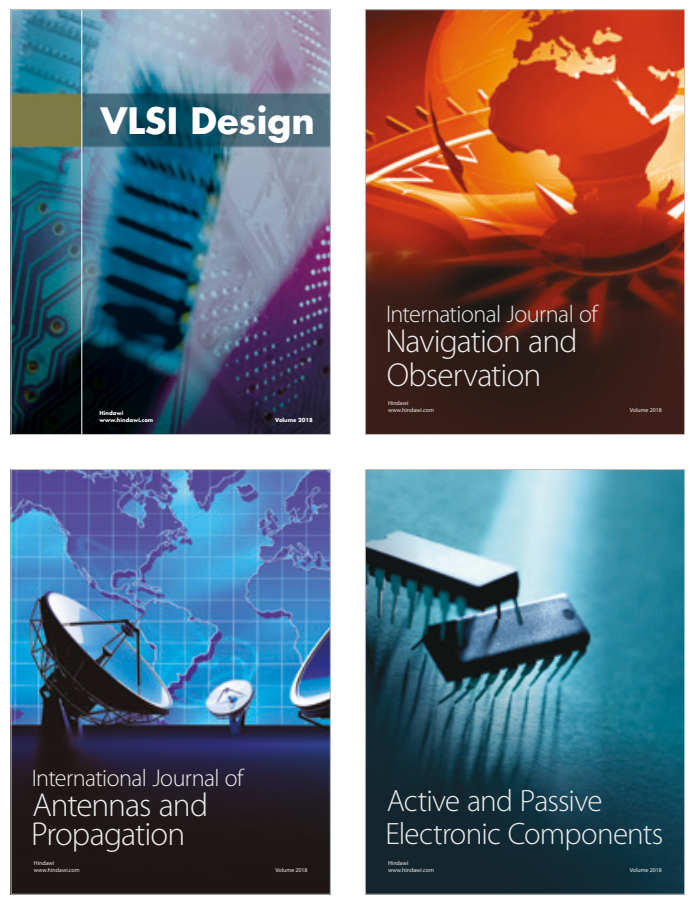
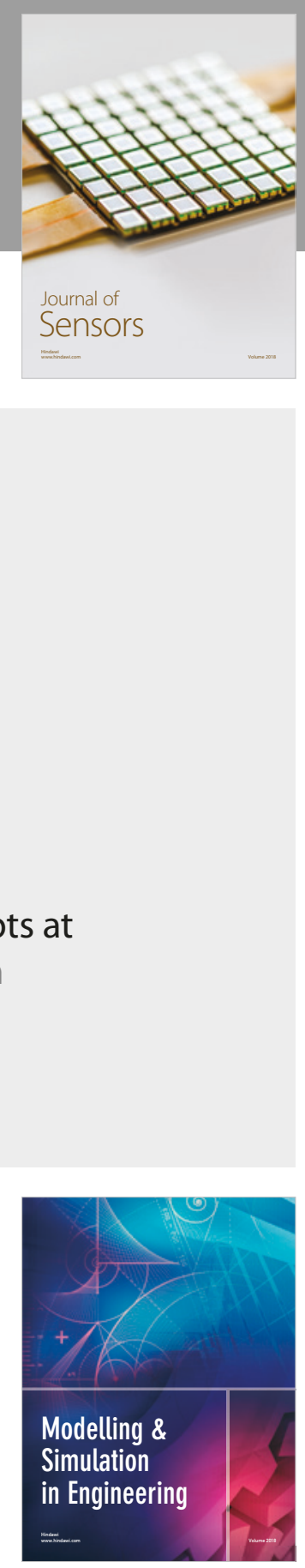

\section{Advances \\ Multimedia}
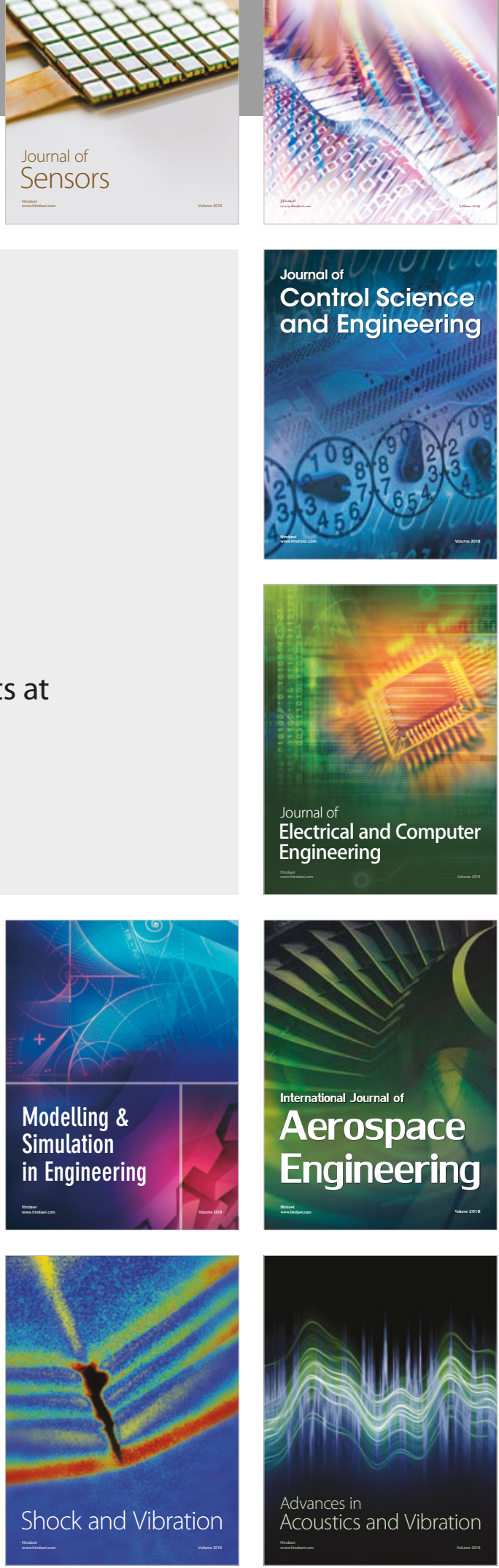